\title{
On the existence of smooth Cauchy steep time functions
}

\author{
E Minguzzi \\ Dipartimento di Matematica e Informatica "U. Dini", Università degli Studi di \\ Firenze, Via S. Marta 3, I-50139 Firenze, Italy. \\ E-mail: ettore.minguzzi@unifi.it
}

\begin{abstract}
A simple proof is given that every globally hyperbolic spacetime admits a smooth Cauchy steep time function. This result is useful in order to show that globally hyperbolic spacetimes can be isometrically embedded in Minkowski spacetimes and that they spit as a product. The proof is based on a recent result on the differentiability of Geroch's volume functions.
\end{abstract}

\section{Introduction}

Let $(M, g)$ be a spacetime, namely a Hausdorff, paracompact, connected, time-oriented Lorentzian manifold, endowed with a $\left(C^{3}\right)$ metric $g$ of signature $(-,+, \cdots,+)$. Since every $C^{r}$ manifold is $C^{r}$ diffeomorphic to a $C^{\infty}$ manifold [6], we shall not bother with the the degree of differentiability of $M$, as we can suppose to have been given a smooth atlas.

On a spacetime a time function $\tau: M \rightarrow \mathbb{R}$ is a continuous function which increases over every causal curve, that is, $x<y \Rightarrow f(x)<f(y)$, where as usual, we write $x<y$ if there is a future-directed causal curve connecting $x$ to $y$. A Cauchy time function is a time function for which the level sets are Cauchy hypersurfaces, namely closed acausal sets intersected exactly once by any inextendible causal curve. A time function $\tau$ is steep if it is continuously differentiable and $-g(\nabla \tau, \nabla \tau) \geq 1$.

The existence of smooth Cauchy time functions in globally hyperbolic spacetimes has been proved through different approaches in [1-3]. It implies that these spacetimes admit a smooth splitting as a product $\mathbb{R} \times S, g=-\beta^{2} \mathrm{~d} t^{2} \oplus h_{t}, \beta: M \rightarrow(0,+\infty)$, where $t$ is the Cauchy time function and $h_{t}$ is a time dependent Riemannian metric over the Cauchy hypersurface $\{t\} \times S$. Recently, Müller and Sánchez [7] proved that the Cauchy time function can be found steep, a fact which implies that $(M, g)$ can be embedded in $N$-dimensional Minkowski spacetime for some $N \geq 2$, and that $\beta$ can be chosen such that $\beta \leq 1$. This result can also be used to express a formula for the Lorentzian distance in terms of the family of time functions [8]. 
In this work we provide a simple proof of the existence of smooth Cauchy steep time functions in globally hyperbolic spacetimes by using some recent results on Geroch's volume functions.

Let $(M, g)$ be a spacetime and let us consider a non-negative continuous function $\varphi$. The Geroch's volume function is

$$
\tau_{\varphi}^{ \pm}(p)=\int_{J^{ \pm}(p)} \varphi d \mu_{g},
$$

where $d \mu_{g}$ is the volume element of $g$.

In a recent joint work with J. Grant and P. Chruściel [2] we proved the following lemma, which basically follows from the differentiability properties of the exponential map.

Lemma 1.1. In globally hyperbolic spacetimes the functions $\tau_{\varphi}^{ \pm}$are continuously differentiable for all continuous compactly supported non-negative functions $\varphi$. The gradient at $p \in M$ is past-directed timelike or vanishing depending respectively on whether $E^{ \pm}(p)$ intersects or not the set $\{q: \varphi(q)>0\}$.

For completeness we mention that the gradient is given by

$$
\left.\nabla_{X} \tau_{\varphi}^{ \pm}=\int_{E^{ \pm}(p)} \varphi J(X)\right\rfloor d \mu_{g},
$$

where $J(X)$ is the Jacobi field obtained solving the Jacobi equation over each generator $\gamma(s)$ of the horismos $E^{ \pm}(p)$, with initial condition $J(0)=X,\left(\frac{d}{d s} J\right)(0)=0$.

In the proof of the main Theorem 1.3 it will be useful to keep in mind the next topological result.

Lemma 1.2. Let $K$ be a compact subset of a topological space and let $\left\{U_{s}\right\}_{s \in S}$ be a locally finite family of subsets. Then $K$ is intersected by at most finitely many elements of the family.

Proof. Let $A_{i}$ be the open (in $K$ ) subset of $K$ made of points which admit a neighborhood which intersects at most $i$ elements of $\left\{U_{s}\right\}_{s \in S}$. Clearly, $A_{i} \subset A_{i+1}$, and $\bigcup_{i} A_{i}=K$. However, this open covering of $K$ admits a finite subcovering which proves that $K=A_{j}$ for some $j$. Since every $p \in K$ admits an open neighborhood $N_{p}$ which intersect at most $j$ elements of the family $\left\{U_{s}\right\}_{s \in S}$, and since $K$ admits a finite subcovering of say, $r$ elements of the form $N_{p_{i}}, K$ is intersected at most by $r j$ elements of the family $\left\{U_{s}\right\}_{s \in S}$.

We are ready to prove the existence of smooth Cauchy steep time functions. The proof uses just the previous Lemmas and Geroch's topological splitting theorem.

Theorem 1.3. Let $(M, g)$ be a globally hyperbolic spacetime. There exists a smooth Cauchy time function $\tau: M \rightarrow \mathbb{R}$ with timelike past-directed gradient $\nabla \tau$ which is steep, namely $-g(\nabla \tau, \nabla \tau) \geq 1$. 
Proof. Just in this proof we shall say that $\tau$ is 'steep' if $-g(\nabla \tau, \nabla \tau)>1$ with the strict inequality. According to Geroch's topological splitting [4,5] there is a continuous Cauchy time function $t: M \rightarrow \mathbb{R}$. The level sets $S_{t}$ are Cauchy hypersurfaces. We are first going to construct a continuously differentiable function $\tau^{-}$(resp. $-\tau^{+}$) over $M$, with past-directed timelike or vanishing gradient, which is steep over $J^{+}\left(S_{0}\right)$ (resp. $J^{-}\left(S_{0}\right)$ ) and such that $\tau^{-}-t>0$ over $J^{+}\left(S_{0}\right)$ (resp. $-\tau^{+}<t$ over $J^{-}\left(S_{0}\right)$ ). Then $\tau^{\prime}=\tau^{-}-\tau^{+}$will be clearly continuously differentiable, steep and Cauchy over $M$.

Next, by [6, Theor. 2.6] $C^{\infty}(M, \mathbb{R})$ is dense in $C^{1}(M, \mathbb{R})$ endowed with the Whitney strong topology [6, p. 35], thus we can find $\tau \in C^{\infty}(M, \mathbb{R})$ which approximates $\tau^{\prime}$, up to the first derivative, as accurately as we want over $M$. In particular, we can find $\tau$ such that $\left|\tau-\tau^{\prime}\right|<1$ and $-g(\nabla \tau, \nabla \tau)>1$, where the former inequality implies that $\tau$ is Cauchy, and the latter inequality implies that $\tau$ is steep.

Let us construct $\tau^{-}$, the plus case being analogous. For every $p \in J^{+}\left(S_{0}\right)$ there is a open neighborhood $V_{p}$ with compact closure contained in $I^{+}\left(S_{-1}\right)$. Let $V_{0}=M \backslash J^{+}\left(S_{0}\right)$, by paracompactness the open covering $\left\{V_{0}\right\} \cup\left\{V_{p}: p \in J^{+}\left(S_{0}\right)\right\}$ admits a locally finite refinement (necessarily countable by Lemma 1.2 and $\sigma$-compactness of $M$ ) and a corresponding partition of unity $\left\{\varphi_{i}, i \geq 0\right\}$, where, defined $U_{j}=\left\{q: \varphi_{j}(q)>0\right\}$ we have for $j \geq 1, \operatorname{supp} \varphi_{j}=\overline{U_{j}} \subset I^{+}\left(S_{-1}\right)$.

Let $K_{i} \subset J^{+}\left(S_{0}\right)$, be a sequence of compact sets such that $K_{0}=\emptyset, \bigcup_{i} K_{i}=J^{+}\left(S_{0}\right)$, $K_{i}=J^{-}\left(K_{i}\right) \cap J^{+}\left(S_{0}\right)$ and

$$
K_{i} \cup \bigcup_{j: U_{j} \cap K_{i} \neq \emptyset} \overline{U_{j}} \subset K_{i+1} .
$$

(The indices $j$ entering the union are finite in number due to Lemma 1.2.) Let $\lambda_{j}$ be a sequence of positive numbers and let $\varphi=\sum_{j=1}^{\infty} \lambda_{j} \varphi_{j}$. Since $\left\{\operatorname{supp} \varphi_{j}\right\}$ is locally finite, $\varphi$ is finite as at any point only a finite number of terms give a non-vanishing contribution. Moreover, $\operatorname{supp} \varphi=\cup_{j=1} \overline{U_{j}} \subset I^{+}\left(S_{-1}\right)$ (it is useful to recall that the closure operator is additive if the union is over a locally finite family [9, Lemma 20.5], and that the closure of a locally finite family is locally finite).

We are going to find a sequence $\lambda_{j}$ such that $\tau^{-}:=\tau_{\varphi}^{-}$is steep over $J^{+}\left(S_{0}\right)$. Observe that

$$
\tau_{\varphi}^{-}=\sum_{j} \lambda_{j} \tau_{\varphi_{j}}^{-}, \quad \nabla \tau_{\varphi}^{-}=\sum_{j} \lambda_{j} \nabla \tau_{\varphi_{j}}^{-} .
$$

By induction suppose that we can find a finite sequence $\lambda_{1}^{i}, \lambda_{2}^{i}, \cdots, \lambda_{n_{i}}^{i},\left(\lambda_{j}^{i}=0\right.$ for $j>n_{i}$ ) such that $\tau^{-}$, defined as above, is steep over $K_{i}$, and satisfies the inequality $\tau^{-}-t>0$ over $K_{i}$. Let $\Lambda_{i}$ be the finite index set such that if $r \in \Lambda_{i}$ then $U_{r} \cap\left(K_{i+1} \backslash \operatorname{Int} K_{i}\right) \neq \emptyset$. We have $U_{r} \cap K_{i-1}=\emptyset$, for otherwise $U_{r} \subset K_{i} \Rightarrow U_{r} \subset \operatorname{Int} K_{i}$, a contradiction. Let $p \in K_{i+1} \backslash \operatorname{Int} K_{i}$, then $p$ belongs to some $U_{r}, r \in \Lambda_{i}$, and hence $E^{-}(p)$ intersects $U_{r}$.

We now replace the constants $\lambda_{r}, r \in \Lambda_{i}, \lambda_{r} \rightarrow \lambda_{r}^{\prime}$, with larger constants chosen in 
such a way that $\tau^{-}$and $\nabla \tau^{-}$get replaced by

$$
\tau_{\varphi}^{-} \rightarrow \tau_{\varphi}^{-}+\sum_{r \in \Lambda_{i}}\left(\lambda_{r}^{\prime}-\lambda_{r}\right) \tau_{\varphi_{r}}^{-}, \quad \nabla \tau_{\varphi}^{-} \rightarrow \nabla \tau_{\varphi}^{-}+\sum_{r \in \Lambda_{i}}\left(\lambda_{r}^{\prime}-\lambda_{r}\right) \nabla \tau_{\varphi_{r}}^{-}
$$

and so that $\tau_{\varphi}^{-}$becomes steep at $p$, and $\tau_{\varphi}^{-}-t$ becomes positive at $p$.

Since $K_{i+1} \backslash \operatorname{Int} K_{i}$ is compact and $\tau^{-}-t$ and $\nabla \tau^{-}$depend continuously on each $\lambda_{j}$, the redefinition of $\lambda_{r}$ and consequently of $\tau^{-}$can be done so as to obtain steepness and positivity of $\tau^{-}-t$ all over $K_{i+1}$. Observe that this redefinition can only increase the value of $\tau^{-}$over $K_{i}$ and that it certainly does not spoil steepness there. Furthermore, it does not change $\tau^{-}$over $K_{i-1}$ thus the built inductive process leads to the desired function $\tau^{-}$.

\section{Acknowledgments}

I thank James Grant and Piotr Chruściel for useful and motivating discussions on this and related problems. This work has been partially supported by GNFM of INDAM.

[1] A. N. Bernal and M. Sánchez. On smooth Cauchy hypersurfaces and Geroch's splitting theorem. Commun. Math. Phys., 243:461-470, 2003.

[2] P. T. Chruściel, J. D. E. Grant, and E. Minguzzi. On differentiability of volume time functions. Ann. Henri Poincaré, 2015. DOI:10.1007/s00023-015-0448-3, arXiv:1301.2909.

[3] A. Fathi and A. Siconolfi. On smooth time functions. Math. Proc. Camb. Phil. Soc., 152:303-339, 2012.

[4] R. Geroch. Domain of dependence. J. Math. Phys., 11:437-449, 1970.

[5] S. W. Hawking and G. F. R. Ellis. The Large Scale Structure of Space-Time. Cambridge University Press, Cambridge, 1973.

[6] M. W. Hirsch. Differential topology. Springer-Verlag, New York, 1976.

[7] O. Müller and M. Sánchez. Lorentzian manifolds isometrically embeddebale in $\mathbb{L}^{N}$. Trans. Am. Math. Soc., 363:5367-5379, 2011.

[8] A. Rennie and B. E. Whale. Generalised time functions and finiteness of the Lorentzian distance. arXiv:1412.5652 v1, 2014.

[9] S. Willard. General topology. Addison-Wesley Publishing Company, Reading, 1970. 